\title{
Editorial
}

\section{Data collection and analysis}

With this issue we are starting national surveys. India is a country of sub continental proportions and our population of 1 billion people makes us the representatives of $16 \%$ of humanity. It is an awesome burden which hitherto we have not handled with adequate responsibility. It is incumbent on all professional organizations in the country including the Association of Plastic Surgeons of India (APSI) to actively participate in data collection of the problems we face and our response to them. For example till recently nobody could tell the correct incidence of clefting per 1000 live births in India---at least not based on large scale population surveys. In the last 3 years APSI has made an effort on a national scale and hopefully we will soon have a reasonable sample survey of the same. It is not complete but it is a beginning. In fact this has been followed up by a state wide survey of Tamilnadu by our Past President Dr. K. Sridhar. Results are awaited. These are good beginnings, but they need to be sustained. In the $21^{\text {st }}$ century information is power and we will ignore this at our peril.

For too long we have heard that we have excellent surgical skills and that we perhaps operate more numbers than most other surgeons in the world. Despite this we do not seem to be in a leadership role even in clinical surgery (basic research is different). In my opinion the sole and very tragic reason is a complete apathy to keep records and do the detailed audit necessary to get data from a wealth of clinical material. It is only in this way that we will get a true picture of what we do and how well we do it, in addition to opening the way for a large number of potential publications.

With this in mind the IJPS is starting a national survey of Plastic Surgery. Included in this issue is a form which is simple to fill and fairly basic in its scope. It will however generate what we believe to be the first ever national sampling of what is done by us as plastic surgeons round the country. The confidentiality of individuals and units will be protected as also the details of their data. What will be published is an overview with national statistics of various procedures performed. If we do this year on year we can then get good trends in our national endeavour---information useful to all---especially for those in policy making and implementation. We hope the readership will give an overwhelming response to this initiative.

India needs to claim its rightful place in the world but we will be able to do this only if you decide to do something about it---so please take the form, fill it and mail it.

Mukund Thatte Editor, Indian Journal of Plastic Surgery 